\title{
Длинноволновое стимулированное излучение и времена жизни носителей в волноводных структурах с квантовыми ямами на основе $\mathrm{HgCdTe}$
}

\author{
(C) В.В. Румянцев ${ }^{*+}$, М.А. Фадеев ${ }^{*+}$, С.В. Морозов ${ }^{*+}$, А.А. Дубинов ${ }^{*+}$, К.Е. Кудрявцев ${ }^{*+}$, \\ А.М. Кадыков" , И.В. Тузов ${ }^{+}$, C.A. Дворецкий॰ , Н.Н. Михайлов ${ }^{\bullet}$, В.И. Гавриленко*+, F. Терре \\ * Институт ффизики микроструктур Российской академии наук, \\ 603950 Нижний Новгород, Россия \\ + Нижегородской государственный университет им. Н.И. Лобачевского, \\ 603950 Нижний Новгород, Россия \\ - Институт фозики полупроводников Сибирского отделения Российской академии наук, \\ 630090 Новосибирск, Россия \\ - Новосибирский государственный университет, \\ 630090 Новосибирск, Россия \\ - Laboratoire Charles Coulomb (L2C), Universite Montpellier II, \\ Montpellier, France \\ E-mail: rumyantsev@ipm.sci-nnov.ru
}

(Получена 27 апреля 2016 г. Принята к печати 10 мая 2016 г.)

\begin{abstract}
Проведены исследования межзонной фотопроводимости и фотолюминесценции в узкозонных волноводных структурах с квантовыми ямами на основе $\mathrm{HgCdTe}$, дизайн которых предназначен для получения длинноволнового стимулированного излучения в условиях оптической накачки. В структурах $n$-типа времена релаксации фотопроводимости достигают нескольких микросекунд, что позволяет наблюдать стимулированное излучение на длине волны 10.2 мкм с низкой пороговой интенсивностью накачки $\sim 100$ Вт/см ${ }^{2}$ при $20 \mathrm{~K}$. В структурах $p$-типа, полученных отжигом для увеличения концентрации вакансий ртути, из-за резкого уменьшения времени жизни относительно безызлучательной рекомбинации Шокли-Рида-Холла не удается наблюдать даже спонтанное излучение из квантовых ям.
\end{abstract}

\section{1. Введение}

В настоящее время компактные полупроводниковые лазеры для инфракрасной (ИК) спектроскопии перекрывают широкий диапазон длин волн в ИК области спектра. В то время как в ближнем ИК диапазоне используются в основном полупроводниковые лазерные диоды, в среднем и дальнем ИК диапазонах превалируют униполярные квантово-каскадные лазеры (ККЛ). Существующая технология ККЛ позволила создать источники излучения в диапазоне длин волн от 3 до 20 мкм, работающие при температурах вплоть до комнатной $[1,2]$. ККЛ используются также для получения терагерцового излучения с частотой до 5ТГц $[3,4]$. Однако эффективность большинства ККЛ падает при длинах волн более 20 мкм, так как в этом диапазоне становится существенным решеточное поглощение в материалах GaAs и InP, которые обычно используются для изготовления ККЛ. В частности, в спектральном диапазоне 20-25 мкм генерацию удалось получить лишь на отдельных длинах волн, соответствующих минимумам фононного поглощения в соответствующих материалах [5-7].

Альтернативным источником излучения в длинноволновой части среднего и дальнем ИК диапазоне являются лазеры на основе халькогенидов свинца $\mathrm{PbSnSe}(\mathrm{Te})$. Coобщается о получении в лазерах этого типа излучения на длинах волн до 46 мкм [8,9], однако их характеристики, в частности выходная мощность, ограничены вследствие сложностей в технологии роста.
В то же время, технология роста другого узкозонного материала $\mathrm{HgCdTe}$ (кадмий-ртуть-теллур, КРТ), развита достаточно хорошо по причине исключительной важности этого материала для производства приемников в ИК области спектра. Молекулярно-лучевая эпитаксия (МЛЭ) позволяет получать высококачественные эпитаксиальные пленки не только на подложках $\mathrm{CdZnTe}$, которые обычно используются для роста $\mathrm{HgCdTe}$, но и на альтернативных GaAs-подложках [10]. Помимо объемных слоев с низкой темновой концентрацией носителей $\left(\sim 10^{14} \mathrm{~cm}^{-3}\right)$ МЛЭ может использоваться для получения гетероструктур с квантовыми ямами (КЯ) на основе узкозонных твердых растворов КРТ [11]. Высокое оптическое качество эпитаксиальных структур на подложках $\mathrm{GaAs}$ (013) подтверждено исследованиями фотопроводимости (ФП) и фотолюминесценции (ФЛ) в среднем и дальнем ИК диапазонах [12-18]. Твердый раствор КРТ позволяет варьировать ширину запрещенной зоны от 0 до 1.6 эВ, а частота оптических фононов в нем сдвинута в низкочастотную область по сравнению c $\mathrm{A}^{\mathrm{III}} \mathrm{B}^{\mathrm{V}}$ материалами. Энергия CdTe-подобных оптических фононов в узкозонных составах КРТ лежит вблизи 20 мэВ [19], а НgТе-подобных еще ниже по энергии около 15 мэВ, благодаря чему получение стимулированного излучения (СИ) в диапазоне длин волн 10-30 мкм, где отсутствует даже двухфононное поглощение, представляется относительно легко достижимым.

Тем не менее исследования стимулированного излучения в КРТ структурах до недавнего времени ограничива- 
лись гораздо более коротковолновой областью [20-24]. В среднем ИК наиболее длинноволновая генерация на длине волны 5.3 мкм при $45 \mathrm{~K}$ была получена в работе [25] еще в 1993 году, однако позднее был сделан вывод, что лазеры на основе КРТ не выдерживают конкуренции с ККЛ в области среднего ИК диапазона. Считается, что создание межзонных лазеров на основе КРТ в длинноволновой части среднего ИК диапазона бесперспективно ввиду сильной оже-рекомбинации в узкозонных материалах, эффективность которой растет с уменьшением запрещенной зоны. Тем не менее, как уже было сказано, в узкозонных твердых растворах на основе халькогенидов свинца и олова удалось добиться лазерной генерации на длинах волн вплоть до 46 мкм. В данных твердых растворах, несмотря на высокую остаточную концентрацию примесей и дефектов (концентрация свободных носителей при $4.2-70 \mathrm{~K}$ превышает $10^{17} \mathrm{~cm}^{-3}$ даже в лучших образцах), ожерекомбинация оказывается подавленной из-за „симметрии“ законов дисперсии электронов и дырок [26,27]. Близость значений эффективной массы электронов и дырок приводит к возникновению энергетического порога для оже-рекомбинации, величина которого порядка ширины запрещенной зоны материала. Аналогичную „симметрию“ можно обеспечить и в гетероструктурах на основе $\mathrm{HgTe} / \mathrm{CdTe}$, в которых при некоторой критической толщине КЯ ширина запрещенной зоны обращается в нуль, а спектр носителей становится „графеноподобным“ [28]. Энергетический спектр гетероструктур с КЯ можно регулировать путем изменения их толщины и содержания кадмия в них. Ряд работ указывает на то, что в таких КРТ структурах оже-рекомбинация подавлена по сравнению с объемными образцами [29], что в конечном счете позволило достичь длинноволнового стимулированного излучения из таких структур при оптической накачке [30].

Необходимым условием для создания длинноволновых источников излучения с токовой накачкой на основе КРТ является возможность получения слоев с проводимостью $p$-типа для реализации $p-n$-перехода. Современная технология МЛЭ позволяет получать как структуры $n$-типа, так и $p$-типа. При этом в материале всегда присутствуют вакансии ртути из-за слабости связи $\mathrm{Hg}-\mathrm{Te}$. При низких концентрациях примесей и дефектов кристаллической решетки структуры на основе КРТ обладают электронным типом проводимости с типичной темновой концентрацией $(2-5) \cdot 10^{14} \mathrm{~cm}^{-3}$. Для получения образцов $p$-типа необходимо легировать структуру акцепторами. Наиболее привлекательной представляется возможность получить структуры $p$-типа с помощью легирования мышьяком, однако в этом направлении существует ряд сложностей, несмотря на активные исследования [31]. В настоящее время наиболее часто образцы $p$-типа получают, используя в качестве акцепторов вакансии ртути. Поскольку вакансии являются по сути дефектами кристаллической структуры, с практической точки зрения важно знать, как повлияет такой метод „легирования“ на время жизни носителей, а также на эффективность люминесценции и возможность получения длинноволнового стимулированного излучения в структурах на основе КРТ. В данной работе были исследованы времена жизни носителей в узкозонных структурах на основе КРТ (с краем фундаментального поглощения в диапазоне 10-30 мкм при температуре жидкого гелия) с различной концентрацией вакансий ртути и соответственно разным типом проводимости.

\section{2. Методика эксперимента}

Для определения времени жизни неравновесных носителей в узкозонных структурах на основе твердых растворов $\mathrm{HgCdTe}$ использовалась прямая методика исследования релаксации сигнала межзонной ФП при возбуждении параметрическим генератором света фирмы Solar (Минск, Беларусь). Этот источник позволяет осуществлять возбуждение короткими импульсами узкополосного излучения с возможностью выбора длины волны в широком диапазоне от 6 до 17 мкм. Сигнал, регистрируемый на цифровом осциллографе „Le Croy“ с верхней граничной частотой 1 ГГц, выводился с помощью 50-омного кабеля и усиливался усилителем с 50-омными входным и выходным сопротивлениями с полосой пропускания 400 МГц. Таким образом, временное разрешение метода определялось длительностью импульса и составляло 7 нс.

Исследования спектров ФЛ проводились в криостате замкнутого цикла, оптически сопряженном с фурье-спектрометром Bruker Vertex 80v. Спектрометр работал в режиме пошагового сканирования, а сигнал ФЛ детектировался с помощью фотоэлектрического HgCdTe приемника Kolmar Technologies D317. За деталями эксперимента можно обратиться к работе [15]. Для накачки использовался Nd : YAG лазер, сопряженный с параметрическим генератором света фирмы Solar (Минск, Беларусь). Этот источник обеспечивает генерацию узкополосных импульсов длительностью 10 нс с перестраиваемой длиной волны в диапазоне 1-2.3 мкм. Диаметр пучка накачки составлял $\sim 1 \mathrm{~cm}$, в то время как типичный размер образцов был $4 \times 8$ мм. Таким образом, возбуждающее излучение полностью покрывало поверхность образца. В зависимости от длины волны возбуждения рассеянное излучение накачки отрезалось с помощью трехмиллиметрового германиевого фильтра, фильтра из арсенида индия или комбинации дихроичных фильтров. Как правило, излучение собиралось с торца образца, поскольку такая геометрия эксперимента оптимальна для наблюдения стимулированного излучения, распространяющегося преимущественно вдоль плоскости КЯ. В некоторых случаях ФЛ собиралась с поверхности образца под углом. Такая геометрия позволяет наблюдать и спонтанное, и стимулированное излучение с хорошим отношением сигнал-шум. 


\section{3. Исследуемые образцы}

Все исследуемые в данной работе структуры были выращены методом МЛЭ на полуизолирующих GaAs (013) подложках с ZnTe и CdTe буферами. Характерная плотность дислокаций в структурах составляла $10^{6} \mathrm{~cm}^{-2}$. Объемные образцы представляли собой эпитаксиальные слои твердого раствора $\mathrm{Hg}_{1-x} \mathrm{Cd}_{x} \mathrm{Te}$ толщиной несколько микрон с долей кадмия $x=0.19-0.23$, окруженные тонкими (100-500 нм) варизонными слоями. Структуры намеренно не легировались. Непосредственно после роста слои обладали проводимостью электронного типа с остаточной концентрацией электронов на уровне $(2-5) \cdot 10^{14} \mathrm{~cm}^{-3}$. Путем отжига структур в атмосфере инертного газа, в ходе которого существенная доля атомов ртути выходит из решетки, создавая, таким образом, высокую концентрацию вакансий ртути [32], для каждой структуры с $n$-типом проводимости были

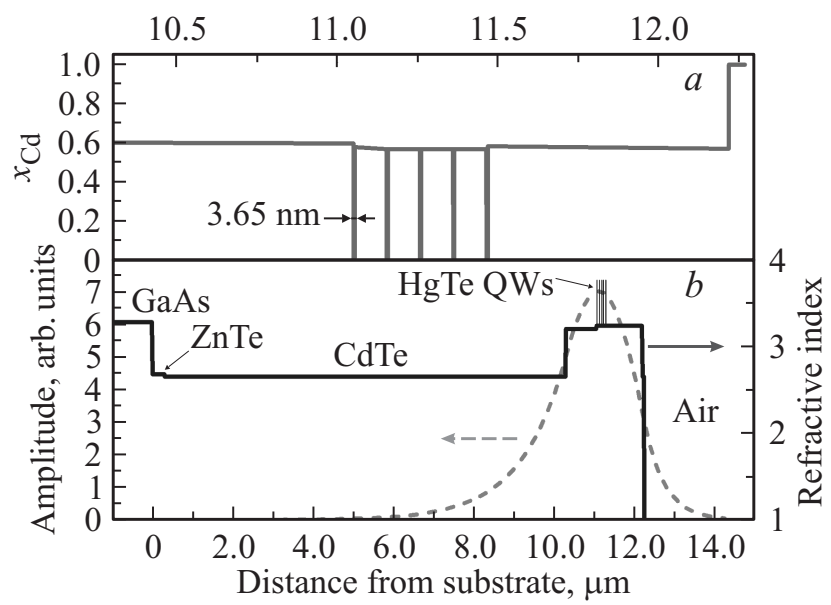

Рис. 1. $a-$ распределение Сd в ВСПСИ № 150120; $b-$ профиль показателя преломления в ВСПСИ № 150120 и расчет локализации моды излучения с длиной волны 8.7 мкм.

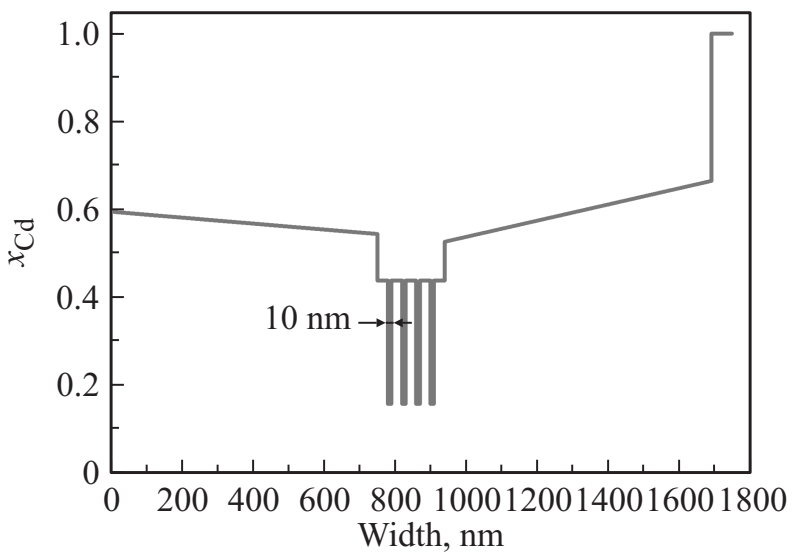

Рис. 2. Распределение Сd в ВСПСИ № 150710 . Начало отсчета выбрано на границе структуры и CdTe буфера (толщиной 10 мкм), выращенного на GaAs (013) подложке (на рисунке не показаны).

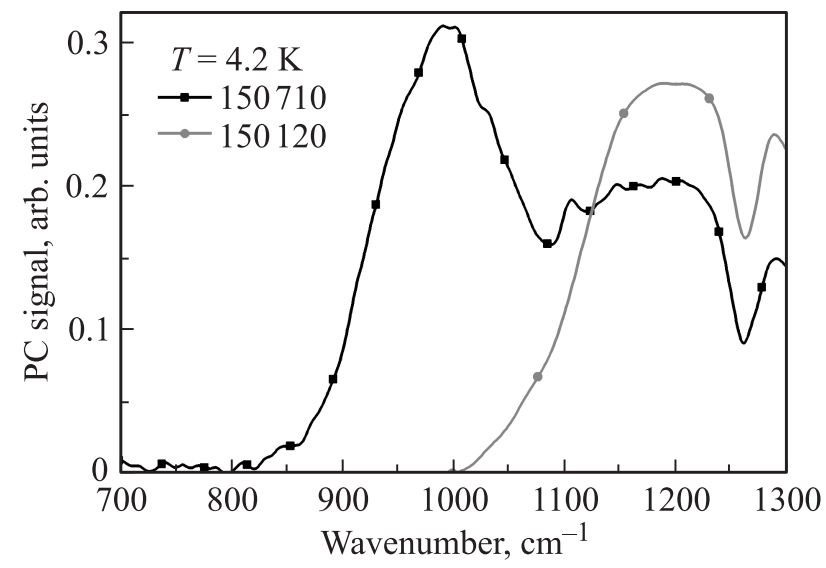

Рис. 3. Спектры фотопроводимости ВСПСИ № 150710 и 150120 в окрестности красной границы межзонного поглощения при температуре жидкого гелия.

получены образцы с тем же значением $x$, но с $p$-типом проводимости. Помимо объемных структур были выращены волноводные структуры для получения стимулированного излучения (ВСПСИ), дизайн которых ориентирован на получение стимулированного излучения в окрестности длины волны $\sim 10$ мкм. Как видно из рис. 1 и 2 , активная область структуры, содержащая несколько (4-5) КЯ, окружена слоями с высокой концентрацией кадмия и низким коэффициентом преломления, который обеспечивает локализацию поля электромагнитной волны в окрестности активной области (см. рис. $1, b)$. Как видно из спектров ФП (рис. 3), красные границы межзонных переходов в ВСПСИ № 150120 и 150710 отличаются незначительно. Таким образом, основное отличие данных структур состоит в различной концентрации вакансий ртути в области КЯ и соответственно типе проводимости.

\section{4. Результаты и обсуждение}

\section{1. Времена релаксации фотоотклика}

Исследования релаксации фотоотклика проводились при температурах жидкого гелия и жидкого азота. В образцах $n$-типа концентрация свободных электронов практически не меняется при переходе от $T=77 \mathrm{~K}$ к $T=4.2 \mathrm{~K}$, поскольку энергии ионизации доноров составляют доли мэВ из-за малой эффективной массы электронов. Напротив, энергии связи акцепторов составляют $8-30$ мэВ $[33,34]$ и при температуре жидкого гелия в структурах $p$-типа происходит „вымораживание“ носителей на уровни акцепторов и концентрация свободных дырок становится ничтожно малой. Тем не менее для всех исследованных образцов было обнаружено, что релаксация носителей в структурах $p$-типа происходит значительно быстрее как при $T=77 \mathrm{~K}$, так и при $T=4.2 \mathrm{~K}$ (см. рис. 4). Таким образом, можно заключить, что существенное укорочение времени жизни носите- 

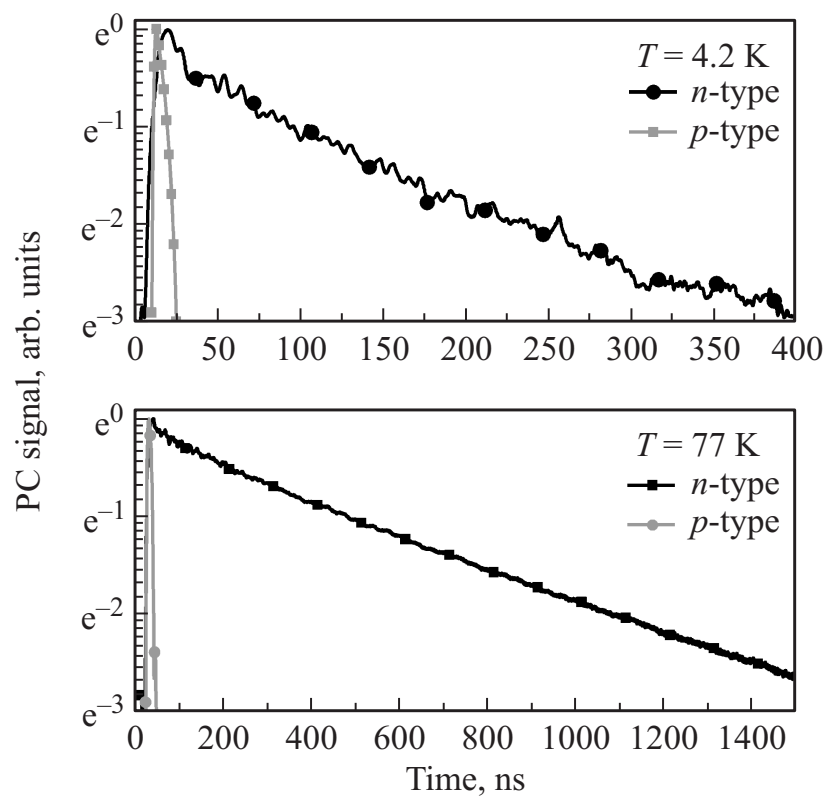

Рис. 4. Кинетика релаксации фотопроводимости образцов $n$ и $p$-типа объемного слоя $\mathrm{HgCdTe}$ с долей кадмия $19 \%$ при температурах жидкого гелия и жидкого азота (длительность возбуждающего импульса 10 нс).
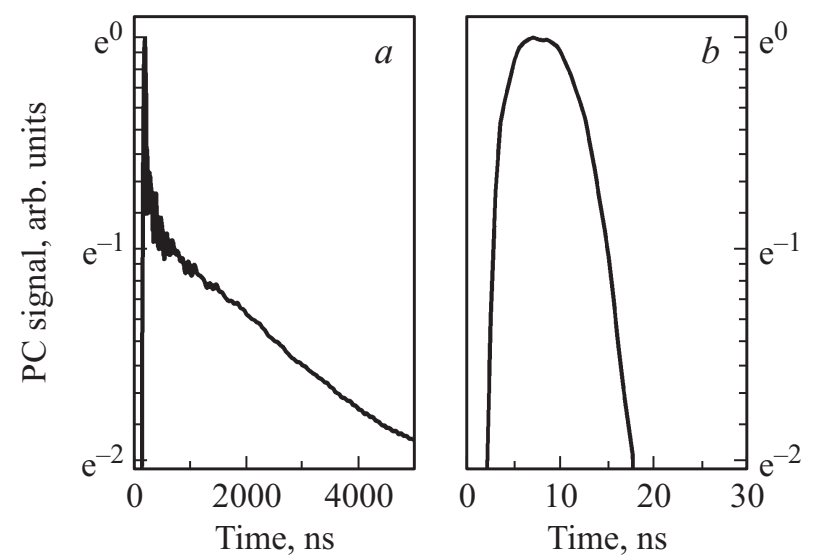

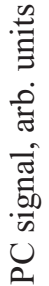

Рис. 5. Кинетика релаксации межзонной фотопроводимости в ВСПСИ № $150120(a)$ и $150710(b)$ при температуре жидкого гелия (длительность возбуждающего импульса $10 \mathrm{Hc}$ ).

лей в образцах $p$-типа, которое наблюдалось для всех исследованных структур, связано, очевидно, с увеличением концентрации вакансий ртути, которое приводит к ускорению рекомбинации носителей по механизму Шокли-Рида-Холла (ШРХ).

Аналогичная картина наблюдается и для ВСПСИ (рис. 5). Для образца № 150710 кинетика фотоотклика фактически повторяла профиль возбуждающего импульca, что говорит о том, что времена жизни составляют менее 7 нс. При этом насыщения рекомбинации ШРХ, которое ранее наблюдалось в некоторых образцах КЯ [16], выращенных с помощью той же технологии, не было обнаружено вплоть до уровней накачки $5 \cdot 10^{23}$ фот/с $\cdot \mathrm{cm}^{2}$.
Напротив, для структуры № 150120 с $n$-типом проводимости наблюдаемое время релаксации фотоотклика составляет 5 мкс, что обеспечивает относительно низкий порог возникновения СИ. Действительно, известно, что для усиления излучения в узкозонных КЯ на основе КРТ необходима двумерная концентрация неравновесных носителей на уровне единиц $10^{11} \mathrm{~cm}^{-2}$ [12]. Принимая длительность импульса накачки за 10 нс, а коэффициент поглощения КЯ - 1\%, легко оценить, что такая концентрация достигается уже при пиковых мощностях возбуждения около $10^{21}$ фот/с · $\mathrm{cm}^{2}$ (в случае, когда времена жизни носителей больше длительности импульса накачки, а возбуждение осуществляется за счет межзонного поглощения в яме).

\section{2. Длинноволновое стимулированное излучение}

Результаты исследований ФЛ в ВСПСИ № 150120 и 150710 согласуются с результатами исследований времени жизни носителей. Так, в структуре № 150120 было получено стимулированное излучение в диапазоне длин волн 10.2-7.5 мкм. На рис. 6 представлены спектры СИ для различных температур. Типичная ширина линии на полувысоте составляет 2-3 мэВ, и, таким образом, ширина линии становится меньше $k T$, начиная с температур $\sim 50 \mathrm{~K}$. Образцы с различных участков структуры дают немного отличающиеся длины волн СИ: $10.2-8.5$ мкм при $18 \mathrm{~K}$; однако, как показывают расчеты, при настолько узких КЯ такой разброс в наблюдаемой энергии межзонных переходов соответствует флуктуациям толщины КЯ на уровне монослоя.

Возникновение узкой линии СИ с увеличением интенсивности накачки сопровождается нелинейным ростом в зависимости интегральной интенсивности ФЛ от мощности накачки. Пороговая плотность мощности накачки

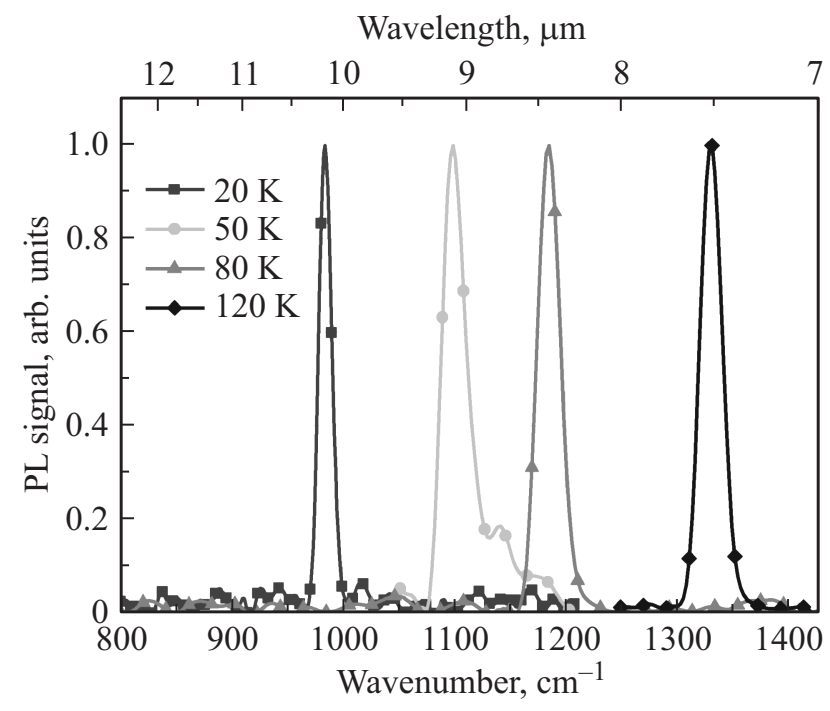

Pис. 6. Спектры стимулированного излучения образца ВСПСИ № 150120 при различных температурах. 
при $20 \mathrm{~K}$ составляет около $120 \mathrm{Bт} / \mathrm{cm}^{2}$ и вырастает до 1 кВт/см ${ }^{2}$ при температуре жидкого азота. Эти данные указаны для возбуждения излучением с длиной волны 2.3 мкм и с хорошей точностью соответствуют оценке, представленной в предыдущем пункте: $10^{21}$ фот/с $\cdot \mathrm{cm}^{2}$ на длине волны 2.3 мкм соответствуют $120 \mathrm{BT} / \mathrm{cm}^{2}$ при диаметре пучка $\sim 1$ см. Отметим, что в данном случае возбуждение является „подбарьерным“: согласно спектру ФП длинноволновый край межзонных переходов в барьерах соответствует длине волны 2.1 мкм и, таким образом, излучение накачки с длиной волны 2.3 мкм поглощается непосредственно в КЯ. Кроме того, несмотря на малую эффективность поглощения возбуждающего излучения в КЯ по сравнению с объемными слоями в барьерах, пороговую интенсивность не удается уменьшить при переходе к более коротковолновой накачке, при которой возбуждающее излучение поглощается и в барьерных слоях. Так, при укорочении длины волны возбуждения до 2 мкм пороговая интенсивность при $20 \mathrm{~K}$ увеличивается до $250 \mathrm{BT} / \mathrm{cm}^{2}$, т. е. возрастает в пересчете на число фотонов. При $80 \mathrm{~K}$ такой резкой разницы не наблюдается - порог возникновения СИ составляет $\sim 1 \kappa \mathrm{KT} / \mathrm{cm}^{2}$ как для накачки с длиной волны 2.3 мкм, так и для 2 мкм. Тем не менее снижения пороговой интенсивности накачки, которую можно было ожидать при эффективной инжекции носителей из барьеров в КЯ, не происходит. При длине волны возбуждающего излучения менее 1.5 мкм вообще не удается наблюдать СИ из КЯ вплоть до интенсивностей накачки 100 кВт/см ${ }^{2}$. Помимо межзонной излучательной рекомбинации в барьерах, которая, как показано в работе [35], существенно увеличивает величину наблюдаемого порога СИ в КЯ, еще одной причиной такого поведения является захват свободных носителей, возникающих в барьерах, на уровни глубоких центров, что затрудняет инжекцию носителей из барьерных слоев, особенно при низких температурах. Подобные глубокие центры с долгоживущими состояниями наблюдались нами ранее в барьерных слоях структур с одиночными КЯ [13].

Учитывая вышесказанное, в дизайн структуры № 150710 были внесены изменения, нацеленные на повышение эффективности инжекции носителей в КЯ: волноводные слои, окружающие активную область, были сделаны варизонными, а барьерные слои с постоянным составом (между КЯ) были изготовлены более тонкими (30 нм) по сравнению с ВСПСИ №150120 (см. рис. 1,2). Несмотря на это, в структуре № 150710, обладающей высокой концентрацией вакансий ртути, не удалось обнаружить не только стимулированного излучения, но и спонтанной ФЛ. На основании этих данных можно сделать вывод, что времена рекомбинации ШРХ существенно короче времен излучательной рекомбинации, изза чего даже в условиях сильной оптической накачки (до $\left.1 \mathrm{MBT} / \mathrm{cm}^{2}\right)$ не происходит насыщения рекомбинации через примесные/дефектные центры и как следствие невозможно получить достаточную концентрацию неравновесных носителей, при которой излучательные процессы будут доминирующими. Следует отметить, что это остается справедливым и при „надбарьерном“ возбуждении. Увеличение мощности „надбарьерной“ накачки приводит лишь к возникновению слабой коротковолновой ФЛ с участием состояний примесей и дефектов в барьерах, а не рекомбинацией в КЯ. Последнее также свидетельствует о том, что трудности в получении концентрации, необходимой для возникновения СИ в КЯ, кроме того, могут быть связаны с эффективным захватом носителей, рожденных в барьерах, на глубокие центры даже при наличии градиента состава, способствующего инжекции носителей в КЯ.

\section{5. Заключение}

Таким образом, в работе были исследованы времена жизни и возможность получения длинноволнового стимулированного излучения в узкозонных гетероструктуpax на основе $\mathrm{HgCdTe}$ с КЯ различного типа проводимости. Измерения кинетики ФП показали, что увеличение концентрации вакансий ртути, за счет которых достигается дырочная проводимость, сильно (не менее чем на 2 порядка) уменьшает время жизни неравновесных носителей, что оказывает отрицательное влияние на излучательные свойства таких структур. В то же время в материале $n$-типа времена жизни достигают нескольких микросекунд, что позволяет получить длинноволновое стимулированное излучение с пороговой интенсивностью накачки около 120 Вт/см². Таким образом, поскольку в условиях сильного возбуждения, когда концентрация неравновесных носителей значительно выше, чем в равновесии, темпы оже-рекомбинации и излучательной рекомбинации слабо зависят от типа проводимости структуры, фактором, определяющим невозможность наблюдения стимулированного излучения из структур p-типа, является рекомбинация Шокли-Рида-Холла.

Работа выполнена при поддержке РФФИ (гранты № 16-32-60172, 16-02-00685) и Министерства образования и науки РФ (грант МК-6923.2016.2). Работа выполнена с использованием оборудования ЦКП ИФМ РАН.

\section{Список литературы}

[1] M.S. Vitiello, G. Scalari, B. Williams, P. De Natale. Opt. Express, 23 (4), 5167 (2015).

[2] M. Bahriz, G. Lollia, A.N. Baranov, R. Teissier. Opt. Express, 23 (2), 1523 (2015).

[3] Q.Y. Lu, S. Slivken, N. Bandyopadhyay, Y. Bai, M. Razeghi. Appl. Phys. Lett., 105 (20), 201102 (2014).

[4] B.S. Williams. Nat. Photon., 1 (9), 517 (2007).

[5] R. Colombelli, F. Capasso, C. Gmachl, A.L. Hutchinson, D.L. Sivco, A. Tredicucci, M.C. Wanke, A.M. Sergent, A.Y. Cho. Appl. Phys. Lett., 78 (18), 2620 (2001).

[6] K. Ohtani, M. Beck. J. Faist. Appl. Phys. Lett. 105 (12), 121115 (2014).

[7] J. Ulrich, J. Kreuter, W. Schrenk, G. Strasser, K. Unterrainer. Appl. Phys. Lett., 80 (20), 3691 (2002). 
[8] K.V. Maremyanin, A.V. Ikonnikov, A.V. Antonov, V.V. Rumyantsev, S.V. Morozov, L.S. Bovkun, K.R. Umbetalieva, E.G. Chizhevskiy, I.I. Zasavitskiy, V.I. Gavrilenko. Semiconductors, 49 (12), 1623 (2015).

[9] L.N. Kurbatov, A.D. Britov, S.M. Karavaev, S.D. Sivachenko, S.N. Maksimovskii, I.I. Ovchinnikov, M.M. Rzaev, P.M. Starik. JETP Lett., 37 (9), 499 (1983).

[10] V.S. Varavin, V.V. Vasiliev, S.A. Dvoretsky, N.N. Mikhailov, V.N. Ovsyuk, Y.G. Sidorov, A.O. Suslyakov, M.V. Yakushev, A.L. Aseev. Proc. SPIE, 5136, 381 (2003).

[11] S. Dvoretsky, N. Mikhailov, Y. Sidorov, V. Shvets, S. Danilov, B. Wittman, S. Ganichev. J. Electron. Mater., 39 (7), 918 (2010).

[12] S.V. Morozov, M.S. Joludev, A.V. Antonov, V.V. Rumyantsev, V.I. Gavrilenko, V.Y. Aleshkin, A.A. Dubinov, N.N. Mikhailov, S.A. Dvoretskiy, O. Drachenko, S. Winnerl, H. Schneider, M. Helm. Semiconductors, 46 (11), 1362 (2012).

[13] S.V. Morozov, V.V. Rumyantsev, A.V. Antonov, A.M. Kadykov, K.V. Maremyanin, K.E. Kudryavtsev, N.N. Mikhailov, S.A. Dvoretskii, V.I. Gavrilenko. Appl. Phys. Lett., 105 (2), 022102 (2014).

[14] S.V. Morozov, V.V. Rumyantsev, A.V. Antonov, K.V. Maremyanin, K.E. Kudryavtsev, L.V. Krasilnikova, N.N. Mikhailov, S.A. Dvoretskii, V.I. Gavrilenko. Appl. Phys. Lett., 104 (7), 072102 (2014).

[15] S.V. Morozov, V.V. Rumyantsev, A.A. Dubinov, A.V. Antonov, A.M. Kadykov, K.E. Kudryavtsev, D.I. Kuritsin, N.N. Mikhailov, S.A. Dvoretskii, V.I. Gavrilenko. Appl. Phys. Lett., 107 (4), 042105 (2015).

[16] S.V. Morozov, V.V. Rumyantsev, A.M. Kadykov, A.A. Dubinov, A.V. Antonov, K.E. Kudryavtsev, D.I. Kuritsin, N.N. Mikhailov, S.A. Dvoretskii, F. Teppe, V.I. Gavrilenko. J. Phys.: Conf. Ser., 647, 012008 (2015).

[17] V.V. Rumyantsev, A.V. Ikonnikov, A.V. Antonov, S.V. Morozov, M.S. Zholudev, K.E. Spirin, V.I. Gavrilenko, S.A. Dvoretskii, N.N. Mikhailov. Semiconductors, 47 (11), 1438 (2013).

[18] V.V. Rumyantsev, S.V. Morozov, A.V. Antonov, M.S. Zholudev, K.E. Kudryavtsev, V.I. Gavrilenko, S.A. Dvoretskii, N.N. Mikhailov. Semicond. Sci. Technol., 28 (12), 125007 (2013).

[19] D.N. Talwar, M. Vandevyver. J. Appl. Phys., 56 (6), 1601 (1984).

[20] A.A. Andronov, Y.N. Nozdrin, A.V. Okomel'kov, A.A. Babenko, V.S. Varavin, D.G. Ikusov, R.N. Smirnov. Semiconductors, 42 (2), 179 (2008)

[21] A.A. Andronov, Y.N. Nozdrin, A.V. Okomel'kov, V.S. Varavin, R.N. Smirnov, D.G. Ikusov. Semiconductors, 40 (11), 1266 (2006).

[22] J. Bleuse, J. Bonnet-Gamard, G. Mula, N. Magnea P. JeanLouis. J. Cryst. Growth, 197 (3), 529 (1999).

[23] J. Bonnet-Gamard, J. Bleuse, N. Magnea, J.L. Pautrat. J. Appl. Phys., 78 (12), 6908 (1995).

[24] C. Roux, E. Hadji J.L. Pautrat. Appl. Phys. Lett., 75 (12), 1661 (1999).

[25] J.M. Arias, M. Zandian, R. Zucca. J. Singh. Semicond. Sci. Technol., 8 (1S), S255 (1993).

[26] J. Dimmock, I. Melngailis, A. Strauss. Phys. Rev. Lett., 16 (26), 1193 (1966).

[27] И.И. Засавицкий. Тр. ФИАН. Оптические и электрические свойства полупроводников, 224, 3 (1993).

[28] B.A. Bernevig, T.L. Hughes, S.C. Zhang. Science, 314 (5806), 1757 (2006).
[29] Y. Jiang, M.C. Teich, W.I. Wang. J. Appl. Phys., 69 (10), 6869 (1991).

[30] S.V. Morozov, V.V. Rumyantsev, A.M. Kadykov, A.A. Dubinov, K.E. Kudryavtsev, A.V. Antonov, N.N. Mikhailov, S.A. Dvoretskii, V.I. Gavrilenko. Appl. Phys. Lett., 108 (9), 092104 (2016).

[31] I.I. Izhnin, S.A. Dvoretsky, K.D. Mynbaev, O.I. Fitsych, N.N. Mikhailov, V.S. Varavin, M. Pociask-Bialy, A.V. Voitsekhovskii, E. Sheregii. J. Appl. Phys., 115 (16), 163501 (2014).

[32] В.С. Варавин, Г.Ю. Сидоров Ю.Г. Сидоров. ЖФХ, 84 (9), 1605 (2010).

[33] Д.В. Козлов, В.В. Румянцев, С.В. Морозов, А.М. Кадыков, В.С. Варавин, Н.Н. Михайлов, С.А. Дворецкий, В.И. Гавриленко, F. Терре. ФТП, 49 (12), 1654 (2015).

[34] I.I. Izhnin, A.I. Izhnin, H.V. Savytskyy, O.I. Fitsych, N.N. Mikhailov, V.S. Varavin, S.A. Dvoretsky, Y.G. Sidorov, K.D. Mynbaev. Opto-Electron. Rev., 20 (4), 375 (2012).

[35] V. Ryzhii, M. Ryzhii, T. Otsuji. J. Appl. Phys., 101 (8), 083114 (2007).

Редактор А.Н. Смирнов

\section{Long-wavelength stimulated emission and carrier lifetimes in $\mathrm{HgCdTe}$ based waveguide structures with quantum wells}

\author{
V.V. Rumyantsev*+, M.A. Fadeev*+, S.V. Morozov*+, \\ A.A. Dubinov*+, K.E. Kudryavtsev*+, \\ A.M. Kadykov*", I.V. Tuzov', S.A. Dvoretskii ${ }^{\bullet}$, \\ N.N. Mikhailov •^, V.I. Gavrilenko*+, F. Teppe" \\ * Institute for Physics of Microstructures Russian \\ Acedemy of Sciences, \\ 603950 Nizhny Novgorod, Russia \\ + Lobachevsky State University of Nizhny Novgorod, \\ 603950 Nizhny Novgorod, Russia \\ - Institute for Semiconductor Physics, Siberian Branch \\ of Russian Acedemy of Sciences, \\ 630090 Novosibirsk, Russia \\ - Novosibirsk State University, \\ 630090 Novosibirsk, Russia \\ - Laboratoire Charles Coulomb (L2C), Universite \\ Montpellier II, \\ Montpellier, France
}

Abstract We present investigation into interband photoconductivity and photoluminescence of narrow gap $\mathrm{HgCdTe}$ based waveguide structures with quantum wells designed to provide long-wavelength stimulated emission under optical pumping. In $n$-type structures carrier lifetimes reach several microseconds and stimulated emission at 10.2 micron wavelength occurs at pumping intensity as low as $\sim 100 \mathrm{~W} / \mathrm{cm}^{2}$ at $20 \mathrm{~K}$. In $p$-type structures, which were obtained by annealing to increase $\mathrm{Hg}$ vacancy concentration, even spontaneous emission has not been detected on account of dramatical decrease of carrier lifetimes due to Shockley-Read-Hall recombination. 